\title{
ON UNIQUENESS AND STABILITY IN GENERALIZED THERMOELASTICITY*
}

BY

\author{
HANY H. SHERIEF
}

Kuwait University

\begin{abstract}
A uniqueness theorem for the equations of generalized thermoelasticity with one relaxation time, derived by Dhaliwal and Sherief [1], is proved. The stability of the null solution in the sense of Liapounov, measured by a suitable norm, is shown. The corresponding equations for a homogeneous isotropic material, derived by Lord and Shulman [2], are considered as a special case.
\end{abstract}

1. Introduction. The equations of generalized thermoelasticity with one relaxation time for a homogeneous isotropic medium were derived by Lord and Shulman [2]. Dhaliwal and Sherief [1] obtained the correspondinig equations for a general anisotropic medium, following a method established by Biot [3] for dealing with the coupled thermoelastic problem. These equations admit the so-called second sound effect in solids; i.e., they predict finite speeds of propagation for heat and displacement disturbances. This is not the situation encountered in coupled thermoelasticity, where an infinite speed of propagation is inherent.

Uniqueness theorems for the equations of generalized thermoelasticity were proved by a number of authors. For the isotropic case, Ignaczak [4] proved a uniqueness theorem for a stress-flex problem, Sherief and Dhaliwal [5] proved a general uniqueness theorem, but their proof relied heavily on the field inequality

$$
-q_{i} \theta_{, i} \geqslant 0 \text { on } B \times[0, \infty),
$$

which was not used directly in the proof of the corresponding classical theorem by Weiner [6]. More recently, Ignaczak [7] established uniqueness by means of an associated conservation law involving higher-order time derivatives. For the anisotropic case, Dhaliwal and Sherief [1] have proved a uniqueness theorem similar to that in [5].

\footnotetext{
${ }^{*}$ Received September 10, 1985.
} 
2. Statement of the problem. We assume that a linear anisotropic thermoelastic material at time $t$ occupies a closed bounded region $B(t)$ of three-dimensional space with sufficiently smooth boundary $\partial B$. The governing equations have the following form [1] in $B \times[0, \infty):$

$$
\rho \frac{\partial^{2} u_{i}}{\partial t^{2}}=\frac{\partial}{\partial x_{j}}\left(c_{i j k l} u_{k, l}\right)-\frac{\partial}{\partial x_{j}}\left(\beta_{i j} \theta\right)+\rho F_{i}
$$

and

$$
\rho c_{E} L\left(\frac{\partial \theta}{\partial t}\right)+T_{0} \beta_{i j} L\left(\frac{\partial^{2} u_{i}}{\partial x_{j} \partial t}\right)=\frac{\partial}{\partial x_{i}}\left(k_{i j} \theta_{. j}\right) .
$$

In the above equations $\rho(\mathbf{x})$ denotes the density, $u_{i}(\mathbf{x}, t)$ are the components of the displacement vector, $c_{i j k l}(\mathbf{x})$ are the elastic moduli, $\theta(\mathbf{x}, t)$ is the temperature deviation above a reference temperature $T_{0}$ chosen such that $\left|\theta / T_{0}\right| \ll 1, F_{i}$ are the components of the external applied force per unit mass, $c_{E}$ is the specific heat for zero deformation, $k_{i j}(\mathbf{x})$ are the components of the conductivity tensor, and $\mathbf{x}\left(x_{1}, x_{2}, x_{3}\right)$ represents a point inside $B(t)$. In Eq. (2.2) we have denoted by $L$ the linear operator

$$
L=1+\tau_{0} \frac{\partial}{\partial t},
$$

where $\tau_{0}$ is the relaxation time.

Equations (2.1) and (2.2) are subject to the initial conditions that at $t=0$ on $B, u_{i}, \dot{u}_{i}$, $\theta$, and $\dot{\theta}$ are prescribed functions of $\mathbf{x}$ and to the boundary conditions that for $t \geqslant 0, \theta$ is prescribed on $\partial B, u_{i}$ is prescribed on a portion $\overline{\partial B_{1}}$ of $\partial B$, while the traction $c_{i j k l} u_{k, l} n_{j}$ is given on $\partial B-\overline{\partial B_{1}}$. We assume also that $u_{i}$ and $\theta$ are continuous in the closure of $B$.

Throughout this paper, the convention is adopted of summing over repeated indices whose range is $1,2,3$.

3. Stability under perturbation of the initial data. Let $\left(u_{i}^{1}, \theta^{1}\right)$ and $\left(u_{i}^{2}, \theta^{2}\right)$ denote two solutions of equations (2.1) and (2.2) with the same force $F_{i}$ and the same boundary conditions but with (possibly) different initial conditions. We shall consider the difference functions

$$
u_{i}=u_{i}^{1}-u_{i}^{2}, \quad \theta=\theta^{1}-\theta^{2},
$$

and take

$$
U_{i}=L u_{i}, \quad \phi=L \theta .
$$

Applying the operator $L$ to (2.1) (with zero $F_{i}$ ) and to the initial and boundary conditions, we see that $\left(u_{i}, \theta\right)$ satisfies

$$
\begin{gathered}
\rho \frac{\partial^{2} U_{i}}{\partial t^{2}}=\frac{\partial}{\partial x_{j}}\left(c_{i j k l} U_{k, l}\right)-\frac{\partial}{\partial x_{j}}\left(\beta_{i j} \phi\right) \text { in } B \times[0, \infty), \\
\rho c_{E} \frac{\partial \phi}{\partial t}+T_{0} \beta_{i j} \frac{\partial^{2} U_{i}}{\partial x_{j} \partial t}=\frac{\partial}{\partial x_{i}}\left(k_{i j} \theta_{. j}\right) \text { in } B \times[0, \infty), \\
U_{i}(\mathbf{x}, t)=0 \text { on } \overline{\partial B_{1}} \times[0, \infty),
\end{gathered}
$$




$$
\begin{gathered}
c_{i j k l} U_{k, l} n_{j}=0 \quad \text { on }\left(\partial B-\overline{\partial B_{1}}\right) \times[0, \infty), \\
\phi(\mathbf{x}, t)=0 \quad \text { on } \partial B \times[0, \infty), \\
U_{i}(\mathbf{x}, 0)=U_{i 0}(\mathbf{x}), \quad \dot{U}_{i}(x, 0)=U_{i 1}(\mathbf{x}) \quad \text { on } B(0), \\
\theta(\mathbf{x}, 0)=\theta_{0}(\mathbf{x}), \quad \phi(\mathbf{x}, 0)=\phi_{0}(\mathbf{x}), \quad \dot{\phi}(\mathbf{x}, 0)=\phi_{1}(\mathbf{x}) \text { on } B(0) .
\end{gathered}
$$

THEOREM. If $\left(U_{i}, \theta\right)$ is a solution of (3.1)-(3.7), then the function

$$
J(t)=T_{0} E(t)+\int_{0}^{t} \int_{B(\eta)} k_{i j} \theta_{. i} \theta_{. j} d \mathbf{x} d \eta,
$$

is a constant independent of time, where

$$
E(t)=\frac{1}{2} \int_{B(t)}\left\{\rho \frac{\partial U_{i}}{\partial t} \frac{\partial U_{i}}{\partial t}+c_{i j k l} U_{i, j} U_{k, l}+\frac{\rho c_{E}}{T_{0}} \phi^{2}+\frac{\tau_{0}}{T_{0}} k_{i j} \theta_{, i} \theta_{. j}\right\} d \mathbf{x},
$$

provided that $k_{i j}$ and $c_{i j k l}$ satisfy the standard symmetry conditions

$$
k_{i j}=k_{j i}, \quad c_{i j k l}=c_{k l i j} .
$$

Proof. From Eq. (3.2), it follows that

$$
\int_{0}^{t} \int_{B(\eta)} \frac{\partial U_{i}}{\partial \eta}\left\{\rho \frac{\partial^{2} U_{i}}{\partial \eta^{2}}-\frac{\partial}{\partial x_{j}}\left(c_{i j k l} U_{k, l}\right)+\frac{\partial}{\partial x_{j}}\left(\beta_{i j} \phi\right)\right\} d \mathbf{x} d \eta=0 .
$$

Integrating by parts, we get

$$
\begin{gathered}
\int_{0}^{t} \int_{B(\eta)} \rho \frac{\partial U_{i}}{\partial \eta} \frac{\partial^{2} U_{i}}{\partial \eta^{2}} d \mathbf{x} d \eta-\int_{0}^{t} \int_{\partial B} c_{i j k l} U_{k, l} \frac{\partial U_{i}}{\partial \eta} n_{j} d S d \eta \\
+\int_{0}^{t} \int_{B(\eta)} c_{i j k l} \frac{\partial^{2} U_{i}}{\partial x_{j} \partial \eta} U_{k, l} d \mathbf{x} d \eta+\int_{0}^{t} \int_{\partial B} \beta_{i j} \frac{\partial U_{i}}{\partial \eta} n_{j} \phi d S d \eta \\
-\int_{0}^{t} \int_{B(\eta)} \phi \beta_{i j} \frac{\partial^{2} U_{i}}{\partial x_{j} \partial \eta} d \mathbf{x} d \eta=0 .
\end{gathered}
$$

The two surface integrals in the last equation vanish by virtue of equations (3.4) and (3.5), respectively. We thus get

$$
\int_{0}^{t} \int_{B(\eta)}\left\{\rho \frac{\partial U_{i}}{\partial \eta} \frac{\partial^{2} U_{i}}{\partial \eta^{2}}+c_{i j k l} \frac{\partial^{2} U_{i}}{\partial x_{j} \partial \eta} \frac{\partial U_{k}}{\partial x_{l}}-\phi \beta_{i j} \frac{\partial^{2} U_{i}}{\partial x_{j} \partial \eta}\right\} d \mathbf{x} d \eta=0 .
$$

Eliminating $\beta_{i j} \partial^{2} U_{i} / \partial x_{j} \partial \eta$ between equations (3.3) and (3.11) yields

$$
\begin{aligned}
\int_{0}^{t} \int_{B(\eta)}\left\{\rho \frac{\partial U_{i}}{\partial \eta} \frac{\partial^{2} U_{i}}{\partial \eta^{2}}+c_{i j k l} \frac{\partial^{2} U_{i}}{\partial x_{j} \partial \eta}\right. & \left.\frac{\partial U_{k}}{\partial x_{l}}+\frac{\rho c_{E}}{T_{0}} \phi \frac{\partial \phi}{\partial \eta}\right\} d \mathbf{x} d \eta \\
& -\frac{1}{T_{0}} \int_{0}^{t} \int_{B(\eta)} \phi \frac{\partial}{\partial x_{i}}\left(k_{i j} \theta_{, j}\right) d \mathbf{x} d \eta=0 .
\end{aligned}
$$


Integrating the last integral by parts and using equation (3.5), gives

$$
\begin{aligned}
\int_{0}^{t} \int_{B(\eta)}\left\{\rho \frac{\partial U_{i}}{\partial \eta} \frac{\partial^{2} U_{i}}{\partial \eta^{2}}+c_{i j k l} \frac{\partial^{2} U_{i}}{\partial x_{j} \partial \eta} \frac{\partial U_{k}}{\partial x_{l}}\right. & \left.+\frac{\rho c_{E}}{T_{0}} \phi \frac{\partial \phi}{\partial \eta}\right\} d \mathbf{x} d \eta \\
& +\frac{1}{T_{0}} \int_{0}^{t} \int_{B(\eta)} k_{i j} \phi_{, i} \theta_{. j} d \mathbf{x} d \eta=0 .
\end{aligned}
$$

Substituting for $\phi$ from the second part of equation (3.1) and using (2.3), we arrive at

$$
\begin{aligned}
\int_{0}^{t} \int_{B(\eta)}\left\{\rho \frac{\partial U_{i}}{\partial \eta} \frac{\partial^{2} U_{i}}{\partial \eta^{2}}+c_{i j k l} \frac{\partial^{2} U_{i}}{\partial x_{j} \partial \eta} \frac{\partial U_{k}}{\partial x_{l}}+\right. & \left.\frac{\rho c_{E}}{T_{0}} \phi \frac{\partial \phi}{\partial \eta}+\frac{\tau_{0}}{T_{0}} k_{i j} \frac{\partial^{2} \theta}{\partial x_{i} \partial \eta} \frac{\partial \theta}{\partial x_{j}}\right\} d \mathbf{x} d \eta \\
& +\frac{1}{T_{0}} \int_{0}^{t} \int_{B(\eta)} k_{i j} \theta_{, i} \theta_{, j} d \mathbf{x} d \eta=0 .
\end{aligned}
$$

Using the symmetry conditions (3.10) and equation (3.9), equation (3.12) yields

$$
\int_{0}^{t} \frac{\partial}{\partial \eta} E(\eta) d \eta+\frac{1}{T_{0}} \int_{0}^{t} \int_{B(\eta)} k_{i j} \theta_{, i} \theta_{, j} d \mathbf{x} d \eta=0
$$

which gives

$$
T_{0} E(t)+\int_{0}^{t} \int_{B(\eta)} k_{i j} \theta_{, i} \theta_{, j} d \mathbf{x} d \eta=T_{0} E(0)
$$

Recalling equation (3.8), equation (3.13) can be written in the final form

$$
J(t) \equiv J(0)
$$

which completes the proof.

Corollary 1. There is at most one solution of equations (2.1) and (2.2) satisfying the prescribed initial and boundary conditions, provided that

(i) $\rho>0, c_{E}>0, T_{0}>0$, and $\tau_{0}>0$,

(ii) $k_{i j}$ and $c_{i j k l}$ satisfy the symmetry conditions (3.10),

(iii) $k_{i j}$ and $c_{i j k l}$ satisfy the positive definiteness conditions

$$
\begin{gathered}
k_{i j} \xi_{i} \xi_{j} \geqslant c_{1} \xi_{i} \xi_{j}, \\
c_{i j k l} \xi_{i j} \xi_{k l} \geqslant c_{2} \xi_{i j} \xi_{i j}
\end{gathered}
$$

for some positive constants $c_{1}$ and $c_{2}$ and for all nonzero tensors $\xi_{i}$ and $\xi_{i j}$.

Proof. As usual, to prove uniqueness the difference functions must satisfy equations (3.1)-(3.5) and the homogeneous counterparts of equations (3.6) and (3.7), namely

$$
\begin{gathered}
U_{i}(\mathbf{x}, 0)=\dot{U}_{i}(\mathbf{x}, 0)=0 \text { on } B(0), \\
\boldsymbol{\theta}(\mathbf{x}, 0)=\phi(\mathbf{x}, 0)=\dot{\phi}(\mathbf{x}, 0)=0 \text { on } B(0) .
\end{gathered}
$$

Using the initial conditions (3.17) and (3.18), it follows from (3.9) that

$$
E(0)=0 .
$$


Equations (3.9), (3.13), and (3.19) give

$$
\begin{aligned}
T_{0} \int_{B(t)}\left\{\rho \frac{\partial U_{i}}{\partial t} \frac{\partial U_{i}}{\partial t}+c_{i j k l} U_{k, l}+\right. & \left.\frac{\rho c_{E}}{T_{0}} \phi^{2}+\frac{\tau_{0}}{T_{0}} k_{i j} \theta_{, i} \theta_{, j}\right\} d \mathbf{x} \\
& +2 \int_{0}^{t} \int_{B(\eta)} k_{i j} \theta_{, i} \theta_{, j} d \mathbf{x} d \eta=0
\end{aligned}
$$

Using conditions (3.16) in (3.20) and taking account of (3.15), we see that all terms in the resulting inequality are nonnegative; this gives

$$
\begin{aligned}
T_{0} \int_{B(t)}\left\{\rho \frac{\partial U_{i}}{\partial t} \frac{\partial U_{i}}{\partial t}+c_{2} U_{i, j} U_{i, j}\right. & \left.+\frac{\rho c_{E}}{T_{0}} \phi^{2}+\frac{\tau_{0}}{T_{0}} c_{1} \theta_{, i} \theta_{, i}\right\} d \mathbf{x} \\
& +2 \int_{0}^{t} \int_{B(\eta)} c_{1} \theta_{, i} \theta_{, i} d \mathbf{x} d \eta=0 .
\end{aligned}
$$

Due to (3.15), all terms on the left-hand side of equation (3.21) are nonnegative; therefore

$$
\frac{\partial U_{i}}{\partial t}=\phi=0 \quad \text { on } \bar{B} \times[0, \infty) .
$$

The first part of equation (3.17) together with (3.22) yields

$$
U_{i}=\phi=0 \text { on } \bar{B} \times[0, \infty) .
$$

Using (3.1), (3.23) may be written as

$$
u_{i}+\tau_{0} \frac{\partial u_{i}}{\partial t}=0, \quad \theta+\tau_{0} \frac{\partial \theta}{\partial t}=0 \quad \text { on } \bar{B} \times[0, \infty) .
$$

As was noted by Ignaczak [7] that equations (3.24) may be written in the equivalent form

$$
\begin{gathered}
u_{i}(\mathbf{x}, t)=u_{i}(\mathbf{x}, 0) \exp \left(\frac{-t}{\tau_{0}}\right) \quad \text { on } \bar{B} \times[0, \infty), \\
\theta(\mathbf{x}, t)=\theta(\mathbf{x}, 0) \exp \left(\frac{-t}{\tau_{0}}\right) \quad \text { on } \bar{B} \times[0, \infty) .
\end{gathered}
$$

Now, using (3.17) and (3.18), the last two equations yield

$$
u_{i}(\mathbf{x}, t)=\theta(x, t)=0 \quad \text { on } \bar{B} \times[0, \infty),
$$

which completes the proof.

Corollary 2. The null solution of equations (2.1) and (2.2) is Liapounov stable in the $J$-norm provided that conditions (3.10), (3.15), and (3.16) are satisfied.

The proof of this corollary follows directly from equation (3.14) and resembles somewhat the linear version of one obtained by Ericksen $[8,9]$ in discussing thermoelastic stability. It also resembles a result of Knops and Payne [10] for the coupled thermoelastic equations.

4. The isotropic case. For this case the elastic moduli $c_{i j k l}$ are given by the relation

$$
c_{i j k l}=\lambda \delta_{i j} \delta_{k l}+\mu\left(\delta_{i k} \delta_{j l}+\delta_{i l} \delta_{j k}\right)
$$


while the components of the conductivity tensor are given by

$$
k_{i j}=k \delta_{i j} \text {, }
$$

where $\lambda$ and $\mu$ are Lamé's constants and $k$ is the coefficient of thermal conductivity.

After some manipulations and using the fact that the tensor $e_{i j}$ defined by

$$
e_{i j}=\frac{1}{2}\left(U_{i, j}+U_{j, i}\right)
$$

is symmetric, the function $J(t)$ in (3.8) can be written in the form

$$
\begin{array}{r}
J(t)=\frac{T_{0}}{2} \int_{B(t)}\left\{\rho \frac{\partial U_{i}}{\partial t} \frac{\partial U_{i}}{\partial t}+\lambda e_{k k}^{2}+2 \mu e_{i j} e_{i j}+\frac{\rho c_{E}}{T_{0}} \phi^{2}+\frac{\tau_{0} k}{T_{0}} \theta_{, i} \theta_{, i}\right\} d \mathbf{x} \\
+k \int_{0}^{t} \int_{B(\eta)} \theta_{, i} \theta_{, i} d \mathbf{x} d \eta=J(0) .
\end{array}
$$

Uniqueness and stability in this case follow from equation (4.4) as before, provided that $\rho, \lambda, \mu, c_{E}, T_{0}, k$, and $\tau_{0}$ are all nonnegative.

\section{REFERENCES}

[1] R. S. Dhaliwal and H. H. Sherief, Generalized thermoelasticity for anisotropic media, Quart. Appl. Math. 38, $1-8(1980)$

[2] H. W. Lord and Y. Shulman, A generalized dynamical theory of thermoelasticity. J. Mech. Phys. Solids 15. 299-309 (1967)

[3] M. A. Biot, Thermoelasticity and irreversible thermodynamics, J. Appl. Phys. 27, 240-253 (1956)

[4] J. Ignaczak, Uniqueness in generalized thermoelasticity, J. Thermal Stresses 2, 171-175 (1979)

[5] H. H. Sherief and R. S. Dhaliwal, A uniqueness theorem and a variational principle for generalized thermoelasticity, J. Thermal Stresses 3, 223-230 (1980)

[6] J. H. Weiner, A uniqueness theorem for the coupled thermoelastic problem, Quart. Appl. Math. 15, 102-105 (1957)

[7] J. Ignaczak, A note on uniqueness in thermoelasticity with one relaxation time, J. Thermal Stresses 5, 257-263 (1982)

[8] J. L. Ericksen, A thermo-kinetic view of elastic stability theory, Internat. J. Solids and Structures 2, 573-580 (1966)

[9] J. L. Ericksen, Thermoelastic stability, Proc. Fifth U.S. Natl. Congr. Appl. Mech., 187--193 (1966)

[10] R. J. Knops and L. E. Payne, On uniqueness and continuous dependence in dynamical problems of linear thermoelasticity, Internat. J. Solids and Structures 6, 1173-1184 (1970) 